\title{
DEVELOPMENT OF SUITABLE MACHINE LEARNING MODEL FOR A CEMENT PLANT CALCINER
}

\author{
Prateek Sharma, M V Ramachandra Rao, Dr B N Mohapatra, A Saxena \\ National Council for Cement and Building Materials, India
}

\begin{abstract}
Cement industry is one of the largest $\mathrm{CO}_{2}$ emitters and continuously working to minimize these emissions. Use of Artificial intelligence (AI) in manufacturing helps in reducing breakdowns/ failures by avoiding frequency of startups, reducing fuel fluctuations which will ultimately reduce carbon footprint. AI offers a new mode of digital manufacturing process that can increase productivity by optimizing the use of assets at the fraction of cost. Calciner is one of the key equipment of a cement plant which dissociates the calcium carbonate into calcium oxide and carbon dioxide by taking heat input from fuel combustion. An AI model of a calciner can provide valuable information which can be implemented in real time to optimize the calciner operation resulting in fuel savings. In this study, key process parameters of continuous operation for a period of 3 months were used to train the various machine learning models and a best suitable model was selected based on metrics like RMSE and $R^{2}$ value. It is found that Artificial neural network is best fitted model for the calciner. This model is able to predict the calciner outlet temperature with high degree of accuracy $(+/-2 \%$ error) when validated against real world data. This model can be used by industries to estimate the calciner outlet temperature by changing the input parameters as it is not based on the chemical and physical process taking place in the calciner but on real world historical data.
\end{abstract}

Keywords- $\mathrm{CO} 2$ emitter, calciner neural network

\section{INTRODUCTION}

Cement industry is one of the largest $\mathrm{CO}_{2}$ emitters and continuously working to minimize these emissions by adopting different levers like reduction in clinker factor, adoption of alternative fuels and raw materials, newer technologies etc. as mentioned in low carbon technology roadmap [1, 2]. Combustion and calcination are two major sources of $\mathrm{CO}_{2}$ emissions in cement manufacturing process. Calciner is one of the major equipment in a cement plant where the calcination of calcium carbonate takes place. Several physical and chemical factors govern the process like thermodynamics and chemical kinetics of the reaction, mass transfer of carbon dioxide and heat transfer between the various phases [3]. The combination of these factors makes the modelling of calciner difficult. Machine learning algorithms learns by training on data generally on historical data to predict or decide without explicitly being programed to do so. Machine learning as shown itself to be a tool capable of forming complex relations between input parameters and desired output [4]. This relationship can't be numerically established or are difficult to establish like that of a calciner. The model discussed in this paper is based on machine learning. Machine learning regression algorithms are powerful tools which can perform better on numerical data with one or more dependent variable and a series of independent variable. Features were extracted and selected accordingly, and exploratory data analysis was conducted. The parameter to be predicted/label was the output temperature of a calciner ( $\mathrm{PC} \mathrm{O/1}$ temp). Various machine learning regression algorithms were trained on the prepared dataset. Comparative analysis of the root mean squared error and $\mathrm{R}^{2}$ score of the algorithms on test dataset was conducted and the best among them was selected for the model namely artificial neural network.

\section{Machine Learning Algorithms}

linear regression: linear regression technique is well know machine learning techniques in which dependent variable is continuous and independent variables are either discrete or continuous. In this model an relationship exists between dependent $(\mathrm{Y})$ and independent variable $(\mathrm{X})$ and is given by the equation $\mathrm{Y}=\mathrm{a}+\mathrm{bX}+\mathrm{c}$

Lasso and ridge regression uses large number of features to reduce problem of over fitting and complexity of models. Lasso regression uses L1 regularization to minimize prediction error for response variable. On the other hand, ridge regression uses L2 regularization and thus can be used when the data set is of multicollinear.

A Random forest regressor uses parallel ensembling technique by fitting several decision trees in parallel. By this model we can predict accuracy and can minimize over-fitting. 

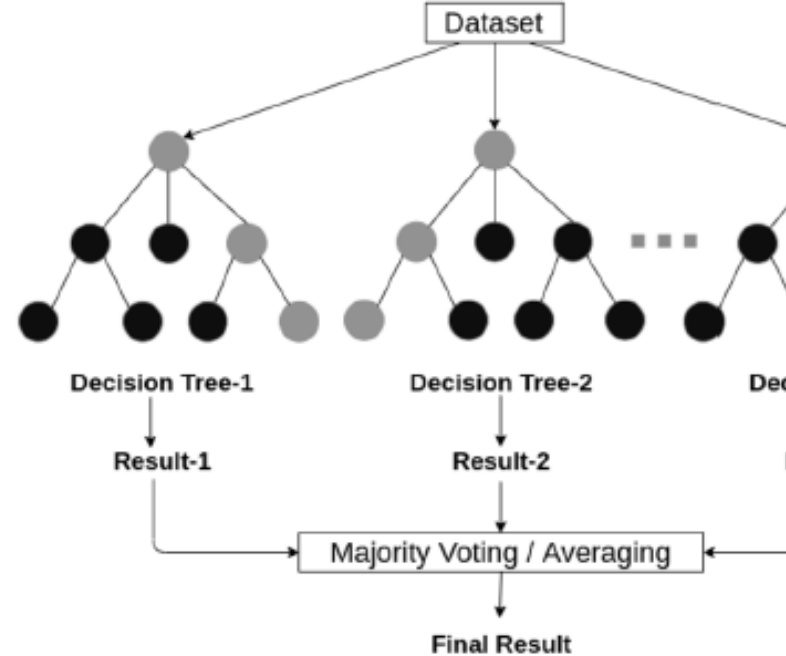

Fig-1: An example of Random forest regressor

Artificial Neural Network: ANN models work in similar way as biological neurons, it consists of 3 layers input, hidden and output layers, each input is modified and multiplied by weight factor. This model can be helpful for learning from large datasets.

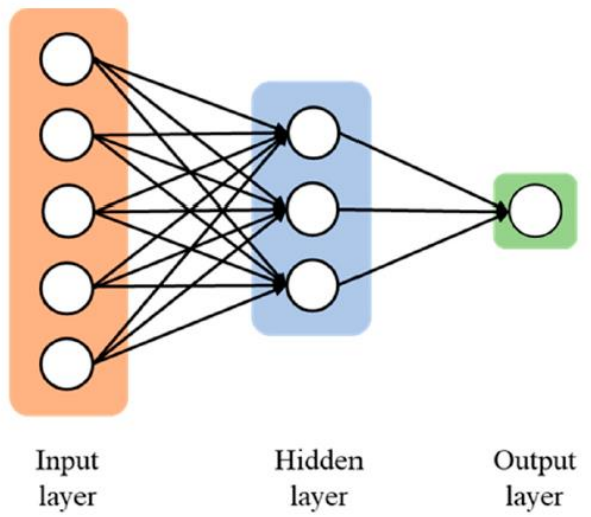

Fig-2: Example of a neural network

\section{MODEL DEPLOYMENT AND RESULT}

The machine learning modeling process comprises of various operations performed from collection of raw data to the implementation of the algorithms to learn. The various subdivisions are listed below:

- Collection of raw data

- Feature Extraction

- Feature Selection

- Exploratory Data analysis

- Data Visualization

- Feature extraction

- Implementation of ML regression algorithms

- Tuning of hyperparameters
- Comparing errors different regression algorithms on test dataset

The input comprises of various parameters which are recorded in the daily log sheet of plants like kiln feed, kiln speed, preheater fan speed, preheater outlet gas temperature, draught, tertiary air and other correlated parameters. Initially the output of the model is outlet temperature of calciner however new outputs can be generated by training on relevant dataset. The raw data was collected for a period of 3 months of continuous operation of a cement production plant. The features were selected which usually corresponds to the working of a calciner. A dataset was prepared of sufficient length to help identify outliers, increase accuracy of the model. Heat map correlation of the dataset was plotted to get a measure of correlation between the features and the label. The features which correlated with the label with a large magnitude were selected while rest were dropped from the feature set. The dataset was divided into training, validation and testing datasets.

\section{Correlation Heatmap}

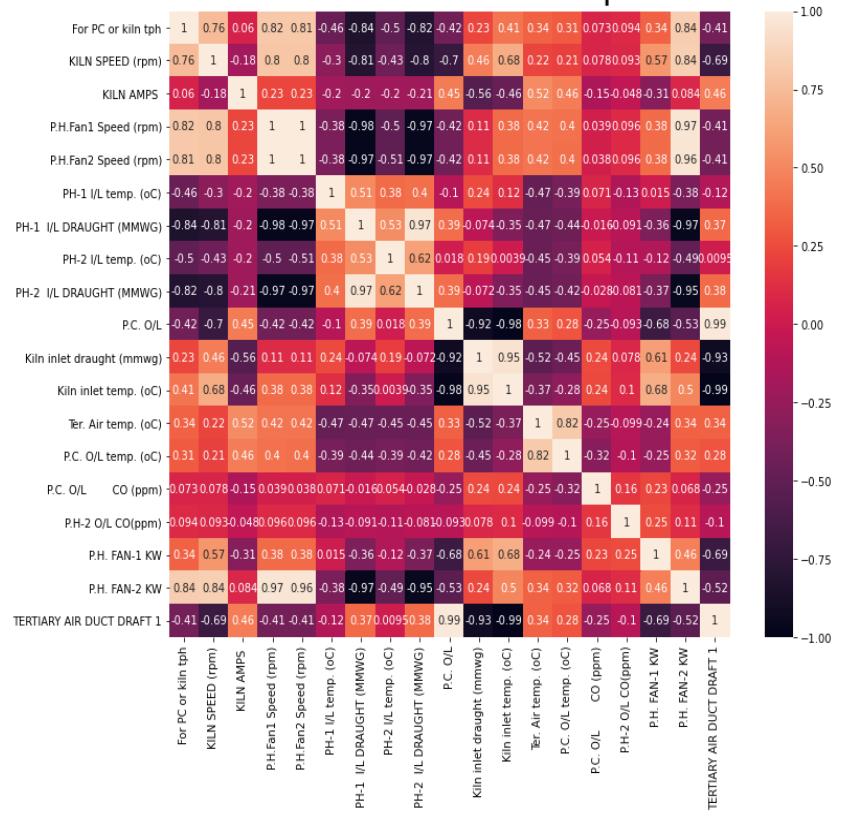

Figure 3: Correlation Heat Map of the dataset

Plant data also includes some data related to breakdown/abnormal operation. So various outliers, missing data points crept into the dataset. These outliers and missing data points made the dataset statistically skewed which in turn makes the prediction misleading. To find outliers, interquartile method was employed and visualized using boxplot shown in fig 2 . 


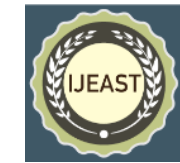

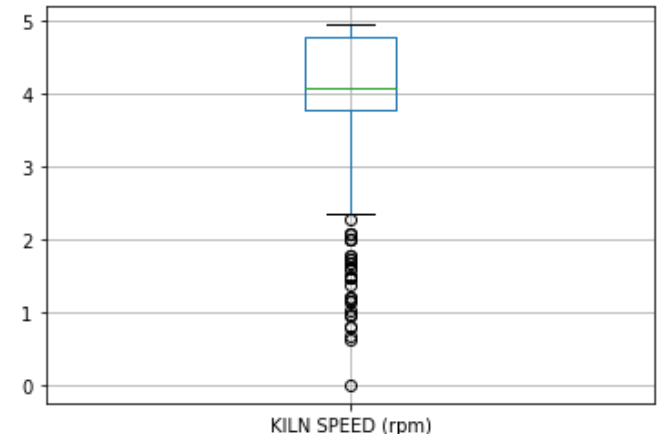

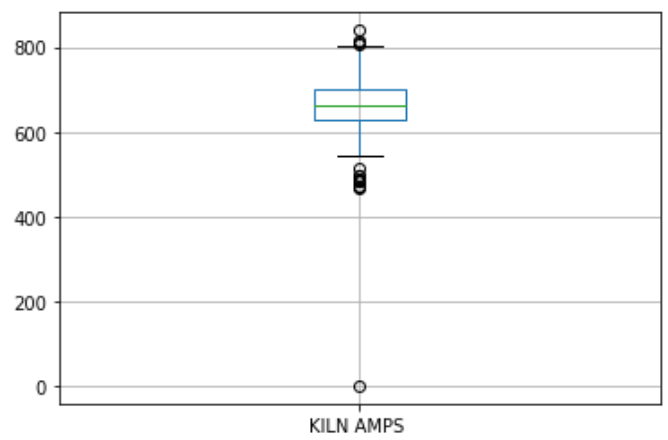

Figure 4: Boxplots of kiln speed and amperage depicting the presence of outliers.

The standard practice to deal with outliers is to either remove them or replace them with a central tendency. The latter was performed with central tendency being median. The fig 3 shows the boxplots of kiln speed and amperage after outliers were replaced with median.
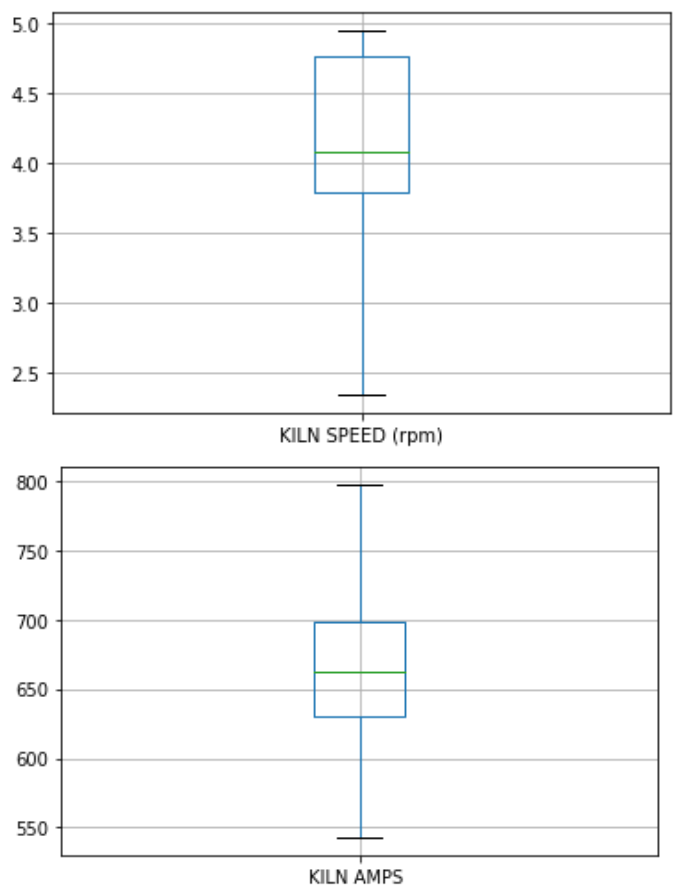

Figure 5: Boxplots of kiln speed and amperage after replacing outliers with median.
A correlation heat map was plotted again (fig4) visualizing the effect of the operation described above.

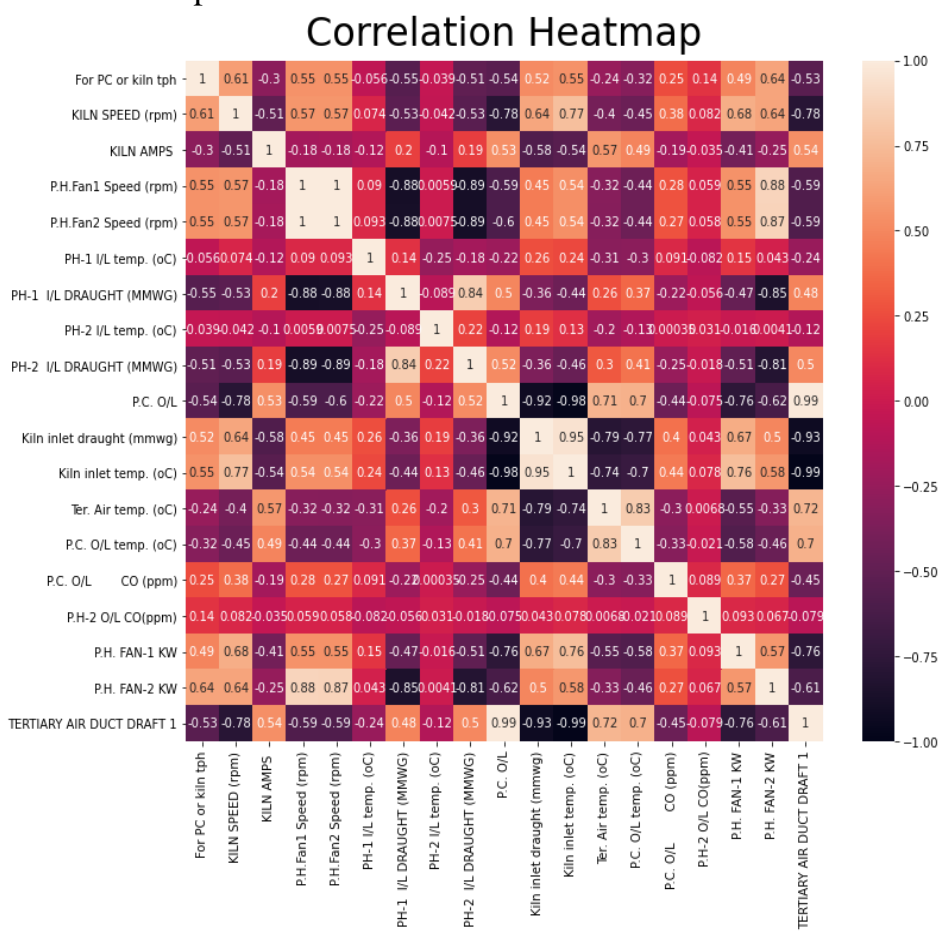

Figure 5: Correlation Heat map of the dataset after replacing outliers with median.

The heat map showed the increase in magnitude and change in nature of correlation between the features and label. The effects due to the presence of outliers in the dataset is established by the heat maps (Fig 1 \& Fig 4). It can be inferred from above heat map that tertiary air temperature and draft, PC outlet draft, preheater 1 and 2 inlet drafts and kiln amps are positively correlated with calciner outlet temperature which also matches with normal cement plant operation [9]. The regression algorithms were trained on the datasets with hyperparameters tuned accordingly to minimize prediction errors on validation dataset.

\section{RESULTS}

The algorithms implemented were tested on test dataset. Root mean squared error and R2 scores were calculated, tabulated (Table 1) and plotted as shown in bar graph (Fig $5)$.

Table 1:Algorithms with their RMS error and $\mathrm{R}^{2}$ score

\begin{tabular}{|c|c|c|}
\hline Algorithms & RMSE & $\mathrm{R}^{2}$ Score \\
\hline ANN & 15.65 & 0.87 \\
\hline $\begin{array}{c}\text { GB Decision } \\
\text { Tree }\end{array}$ & 16.77 & 0.83 \\
\hline
\end{tabular}


International Journal of Engineering Applied Sciences and Technology, 2021

Vol. 6, Issue 3, ISSN No. 2455-2143, Pages 194-198

Published Online July 2021 in IJEAST (http://www.ijeast.com)

\begin{tabular}{|c|c|c|}
\hline XPG Boost & 18.24 & 0.81 \\
\hline $\begin{array}{c}\text { Linear } \\
\text { Regression }\end{array}$ & 18.37 & 0.80 \\
\hline $\begin{array}{c}\text { Ridge } \\
\text { Regression }\end{array}$ & 18.37 & 0.80 \\
\hline $\begin{array}{c}\text { Lasso } \\
\text { Regression }\end{array}$ & 18.47 & 0.80 \\
\hline $\begin{array}{c}\text { Random } \\
\text { Forest }\end{array}$ & 19.43 & 0.78 \\
\hline $\begin{array}{c}\text { Basic } \\
\text { Decision Tree }\end{array}$ & 26.51 & 0.59 \\
\hline
\end{tabular}

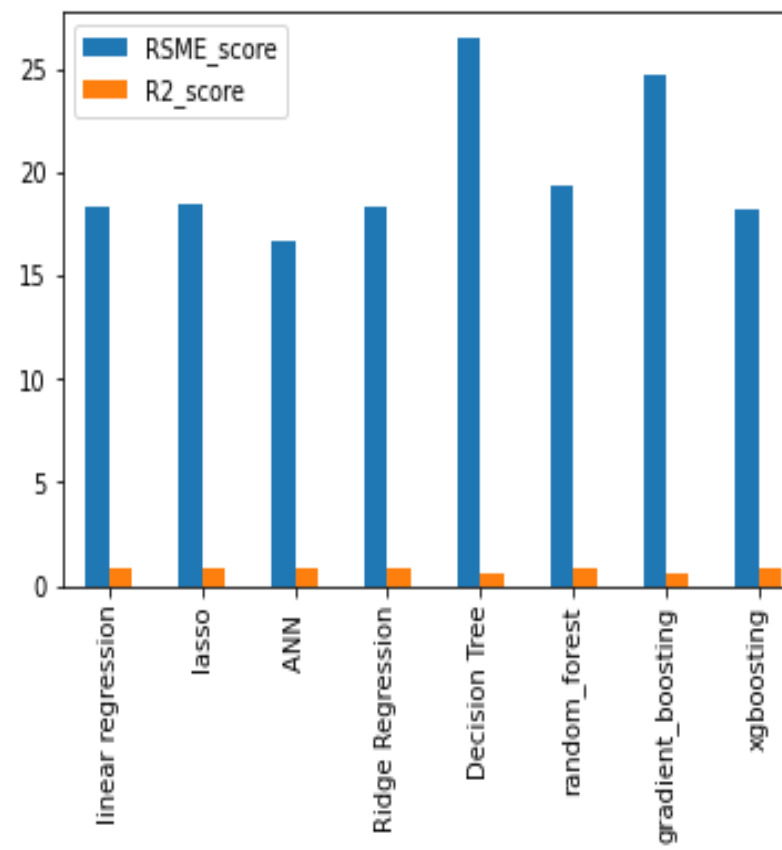

Figure 6: Bar graph of RMSE and $\mathrm{R}^{2}$ score of algorithms on test dataset

The neural network performed best among the algorithms as indicted by highest $\mathrm{R}^{2}$ value and lowest RMSE value. The table 2 shows the calciner outlet temperature of the ANN

Table 2: Calciner outlet temperature predictions of ANN

\begin{tabular}{|c|c|c|}
\hline $\begin{array}{c}\text { Predictions } \\
\left({ }^{\circ} \mathrm{C}\right)\end{array}$ & $\begin{array}{c}\text { True } \\
\text { Values } \\
\left({ }^{\circ} \mathrm{C}\right)\end{array}$ & $\begin{array}{c}\text { \% Absolute } \\
\text { Error }\end{array}$ \\
\hline 975 & 973 & 0.28 \\
\hline 895 & 892 & 0.33 \\
\hline 978 & 976 & 0.50 \\
\hline 946 & 950 & 0.33 \\
\hline 897 & 903 & 0.50 \\
\hline
\end{tabular}

\begin{tabular}{|c|l|l|}
\hline 894 & 910 & 1.66 \\
\hline 886 & 889 & 0.26 \\
\hline 1001 & 994 & 0.72 \\
\hline 960 & 974 & 1.35 \\
\hline
\end{tabular}

\section{CONCLUSION}

Modelling of calciner through machine learning is possible. The ANN model was able to predict the outlet temperature with high degree accuracy (+/- $2 \%$ error) when validated against real world data. The model was able to correlate parameters whose association can't be established through the other models as of now. It can be used to provide an estimate for the plant as it is based on the actual data of a working plant. This model can be trained on relevant data to generate desired outputs. Machine learning can be used to simulate other processes in the cement plant which can give predictions in consonance with real world values. It can help design new plants considering data generated from the model for increased productivity. This model can be used by industries to estimate the outlet temperature by changing the input parameters as it is not based on the chemical and physical process taking place in the calciner but on real world historical data. Being trained on real world data, it inherently provides the model with the ability to account for real world losses like heat loss, efficiency of the system etc. to provide with a prediction which is close to the true value. This models also helps us identify the parameters to be correlated which are usually not associated with the process of calcination thus enabling better utilization of assets to increase productivity.

\section{REFERENCE}

[1] World Business Council for Sustainable Development. Low Carbon Technology Roadmap for the Indian Cement Sector: Status Review (2018

[2] International energy agency. Technology Roadmap LowCarbon Transition in the cement industry. 2018

[3] Y.C. Zhang, Shen-Xue \& Shao, Shuai \& Chen, Yu \& Liu, Su-Ling \& Zhang, Shu-Shen. Aspen Plus-based simulation of a cement calciner and optimization analysis of air pollutants emission. Clean Technologies and Environmental Policy. 13. 459-468. 10.1007/s10098-0100328-y. . (2011).

[4] T. Wuest, D. Weimer, C. Irgens, K.-D. Thoben. Machine learning in manufacturing: advantages, challenges, and applications. Production \& Manufacturing Research. 4 (2016) 23-45. 
[5] J.C. Schrag, F.K. Jäger, H. Köhne, F. Becker. Modelling of the rotary kiln process with Artificial Neural Networks (ANN). 54 (2005) 320-6..

[6] A.K. Pani, V.K. Vadlamudi, H.K. Mohanta. Development and comparison of neural network based soft sensors for online estimation of cement clinker quality. ISA Transactions. 52 (2013) 19-29.

[7] Ö. Çepelioğullar Mutlu, I. Mutlu, S. Yaman, H. Acma. A study to predict pyrolytic behaviors of refuse-derived fuel (RDF): Artificial neural network application. Journal of Analytical and Applied Pyrolysis. 122 (2016).

[8] M. Nakhaei, D. Grévain, L.S. Jensen, P. Glarborg, K. Dam-Johansen, H. Wu. NO emission from cement calciners firing coal and petcoke: A CPFD study. Applications in Energy and Combustion Science. 5 (2021) 100023.. 